\title{
Transcontinentales
}

Sociétés, idéologies, système mondial

\section{Théories et pratiques de la réforme dans le monde arabe}

Réformisme graduel ou transition accélérée?

Theory and Praxis of Reform in the Arab World: Gradual Reform or Accelerated

Transition?

Jean-Noël Ferrié

\section{OpenEdition}

Journals

Édition électronique

URL : http://journals.openedition.org/transcontinentales/259

DOI : 10.4000/transcontinentales.259

ISBN : 978-2-8218-1410-3

ISSN : 1775-397X

Éditeur

Editions de la maison des sciences de l'homme

Édition imprimée

Date de publication : 31 décembre 2005

Pagination : $35-49$

ISBN : 2200-92168-3

ISSN : 1950-1684

Référence électronique

Jean-Noël Ferrié, «Théories et pratiques de la réforme dans le monde arabe », Transcontinentales [En

ligne], 1 | 2005, document 3, mis en ligne le 03 février 2011, consulté le 08 septembre 2020. URL

http://journals.openedition.org/transcontinentales/259; DOI : https://doi.org/10.4000/

transcontinentales.259

Ce document a été généré automatiquement le 8 septembre 2020

Tous droits réservés 


\title{
Théories et pratiques de la réforme dans le monde arabe
}

\author{
Réformisme graduel ou transition accélérée? \\ Theory and Praxis of Reform in the Arab World: Gradual Reform or Accelerated \\ Transition?
}

Jean-Noël Ferrié

1 La réforme politique du monde arabe est à l'ordre du jour, avec une acuité particulière, depuis le 11 septembre 2001. L'intérêt porté à la démocratisation des régimes autoritaires de la région est, cependant, plus ancien. Dans les années 1980 et, plus encore, dans les années 1990, ces régimes ont, en effet, été soumis à des pressions portant sur le respect des droits de l'homme. C'était là le surgeon oriental de l'esprit d'Helsinki qui consistait à considérer certains aspects de la vie interne des États comme relevant des relations internationales. En 1995, le «processus » de Barcelone ajouta la bonne gouvernance et la promotion de la société civile aux critères entrant dans l'évaluation des relations interétatiques entre pays du nord et du sud de la Méditerranée. Les politiques de la Banque mondiale et du Fonds monétaire international ont suivi le même cours. Jusqu'à présent, cependant, ces critères étaient incidents: ils intervenaient comme condition dans la conclusion d'accords ayant d'autres fins. Ils relevaient de la conditionnalité politique affectant des programmes d'aide ou de coopération économiqu ${ }^{1}$. Toutefois, la conditionnalité politique n'était ni la seule ni, surtout, le principal paramètre, puisque les aides étaient d'abord liées à la libéralisation économique, considérée comme une étape vers la libéralisation politique $^{2}$. En ce sens, adopter des politiques de libéralisation, fort partielles au demeurant $^{3}$, pouvait passer pour une forme d'engagement dans un processus de démocratisation. Plusieurs pays ont usé de ce subterfuge, notamment l'Égypte et la Tunisie $^{4}$. Cependant, ce qui est demandé, aujourd'hui, est autre, puisqu'il s'agit de conduire une réforme politique au principal et non plus de manière incidente.

2 La raison de cette demande provient d'une mutation radicale de l'analyse américaine. Les États-Unis avaient adopté - disons depuis la guerre froide - une optique 
metternichienne, où ce qui importait le plus, à leurs yeux, était la stabilité des gouvernements, et donc des relations stratégiques, indépendamment des rapports que les gouvernants, qu'ils soutenaient, entretenaient avec leurs peuples. Washington considère maintenant que la stabilité et la fiabilité des gouvernements est étroitement dépendante de la satisfaction des gouvernés et, plus largement, que la stabilité des gouvernants ne préjuge pas de la fiabilité des peuples. En ce sens, un allié loyal des États-Unis peut être le porteur sain, en quelque sorte, des « pathologies » qui causèrent les attentats du 11 septembre 2001. Une partie des conservateurs américains sont ainsi persuadés que le mal existe en profondeur, indépendamment de la bonne volonté ou du loyalisme des gouvernants ${ }^{5}$. Cette idée grippe le mécanisme bien huilé qui, depuis la guerre froide, permettait aux régimes alliés des États-Unis d'échanger la bienveillance des Américains sur leurs pratiques autoritaires contre l'alignement sur les positions diplomatiques de Washington. Ce revirement de doctrine n'a toutefois pas entraîné un revirement aussi radical dans les pratiques. On aurait ainsi une certaine peine à déduire de la guerre d'Irak, l'autre volet de la politique américaine en faveur de la démocratisation des régimes autoritaires. Celle-ci se fonde encore pour l'essentiel, sur le gradualisme, c'est-à-dire sur la nécessaire antériorité des modifications structurelles et des réformes «step by step » sur les transformations politiques déterminantes. En fait, il semble que l'on soit ici dans une stratégie mixte, mêlant au niveau régional une constante gradualiste, et des tentatives plus ou moins abouties destinées à obtenir des transformations politiques rapides.

Du point de vue de la théorie sociologique, l'approche gradualiste n'a rien à voir avec l'approche qui prône et rend compte des transformations dans le court terme. La première est fondée sur une conception structurelle de la démocratie, selon laquelle le changement social n'est que la conséquence d'évolutions de fond, touchant à la fois aux conditions économiques et sociales et à la culture. La seconde considère, au contraire, que les changements sont liés à l'action réflexive des personnes ${ }^{6}$. Nous avons donc, d'un côté, une conception relevant du holisme sociologique et, de l'autre, une conception relevant de l'individualisme méthodologique. Si ces deux sociologies s'avèrent tranchées dans leurs fondements, elles paraissent, néanmoins, mêlées au fil des analyses : on attribuera facilement la faiblesse de la participation politique - et « donc » de la démocratie - dans le monde arabe aux déficiences du système éducatif, tout en expliquant l'absence de manifestations par la crainte de la répression. Ce faisant, on perd de vue que les deux explications ne sont pas cumulables, puisque que la crainte de la répression peut bloquer des manifestants disposant d'un bon niveau d'éducation, et que s'attacher à l'une c'est, en bonne logique, constater l'inutilité de l'autre. En même temps, les pratiques de la réforme mêlent le volontarisme en Irak et le gradualisme en Égypte, et donnent lieu à des stratégies régionales mixtes qui peuvent paraître cohérentes, de sorte que l'on est amené derechef à user simultanément d'explications holistes et d'explications individualistes, au principal bénéfice du gradualisme, comme théorie et comme pratique. À l'encontre de cette tendance, je voudrais soutenir que le gradualisme est une doctrine sociologique erronée et une pratique contre-productive, et qu'il convient de lui substituer résolument une approche analytique dérivée de la théorie des transitions démocratiques, et attentive aux événements par lesquels se dérèglent les régimes autoritaires et par lesquels ces dérèglements peuvent ou ne peuvent pas aboutir à un mouvement social.

4 Je présenterai d'abord le gradualisme, puis la théorie des transitions, en insistant ensuite sur les fortes différences séparant ces approches et sur le caractère erroné de la 
première. Dans un quatrième temps, je soulignerai, à propos de l'exemple américain, le caractère insatisfaisant des stratégies mixtes dominées par le gradualisme, avant d'analyser comment l'évolution actuelle du Liban permet de considérer différemment les mécanismes à l'œuvre. Dans cette perspective, je soulignerai enfin l'intérêt d'une analyse dérivée de la théorie des transitions, en insistant sur la nécessité de s'intéresser aux dynamiques de déclenchement de ces transitions.

\section{Le gradualisme}

On peut décrire rapidement le gradualisme en disant qu'il consiste à ménager la chèvre et le chou, à respecter le pouvoir des gouvernants tout en démocratisant, et à démocratiser tout en évitant de favoriser les islamistes ${ }^{7}$. Sans doute est-ce ici qu'apparaitt l'idée selon laquelle le développement de la société civile pourrait aider à l'apparition d'une opinion publique concernée, active et pacifique, poussant les gouvernants à la libéralisation sans pour autant faire force commune avec les islamistes. On retrouve la croyance, profondément ancrée dans la philosophie des Lumières, selon laquelle la meilleure façon de lutter contre l'obscurantisme religieux (auquel on assimile implicitement ou explicitement l'islamisme) consiste à s'adresser à la raison des gens, à leurs sentiments sociables, etc. En revanche, le mécanisme d'ensemble se fonde (au mieux) sur une lecture biaisée de Tocqueville. Celui-ci, on le sait, remarque que les «associations politiques » et les " associations civiles » vont de pair. Toutefois, il insiste sur le fait que les associations civiques découlent de l'existence d'associations (et de libertés) politiques ${ }^{8}$. Peut-on faire le pari que ce mécanisme puisse s'inverser et que les associations civiles puissent créer une forme d'association politique? Certainement pas, puisque si la liberté politique permet aux associations civiles de se développer et d'exercer une contrainte sur les associations politiques, l'absence de liberté politique entrave leur développement autonome et ne leur permet pas d'exercer une influence sur la sphère politique. Qui plus est, ces associations civiles s'avèrent tributaires des pouvoirs en place quant à leur existence même. On ne peut donc pas espérer transformer les régimes autoritaires en soutenant les sociétés civiles ; d'abord, parce que la plupart des associations de développement ou des associations caritatives, qui représentent la très grande majorité des associations, sont, pour leur fonctionnement, étroitement dépendantes des régimes en place, et ne poursuivent pas au demeurant d'objectifs politiques; ensuite, parce que les associations de plaidoyer notamment les associations de défense des droits de l'homme - regroupent des élites dénuées de base sociale et dont les préoccupations ne sont pas dominantes au sein de la sociétés.

6 La solution gradualiste comprend, certes, des incitations financières provenant des organisations gouvernementales, des institutions étatiques de coopération des pays développés et de l'Union européenne. Ces incitations vont de l'aide aux institutions de la société civile, c'est-à-dire d'une aide au développement affectée à des ONG (ou devant transiter par elles, ou les impliquant), aux sanctions financières positives, ce que l'on nomme la "conditionnalité politique » et qui consiste à conditionner des subventions au respect de certains principes démocratiques (dont les droits de l'homme) ${ }^{10}$. Ces incitations bénéficient,toutefois, autant aux gouvernants qu'aux associations civiles, dans la mesure où elles viennent pallier les insuffisances des budgets publics dans les secteurs laissés en déshérence par l'État. On a constaté, depuis deux décennies, que les 
associations investissaient des secteurs sociaux que l'État n'était plus à même de gérer et l'on a craint qu'ils ne soient désormais pris en charge par des institutions caritatives islamistes. On a cependant négligé de noter que nombre d'associations caritatives et de développement étaient, d'une manière ou d'une autre, liées à l'État, et que les budgets d'aide et de développement prodigués par les agences occidentales de coopération venaient en quelque sorte compléter le budget de l'État et assurer des services répondant aux attentes de la population. En ce sens, on peut imaginer que le dispositif « aide internationale + associations » œuvre en faveur des gouvernants, en atténuant la pression de la demande sociale plutôt qu'en favorisant son autonomie. Sans doute estce là le dilemme du gradualisme qui entend aider au développement tout en impulsant des réformes substantielles dans leur objectif.

7 Certes, on peut aussi envisager cette efficacité de l'aide comme un moyen de pression, dès lors qu'elle s'avère indéniablement utile aux gouvernants, mais on doit aussi considérer les limites que cette pression permet. Dans la mesure où les gouvernants l'utilisent pour raffermir leur pouvoir, on voit mal pourquoi ils accepteraient, en contrepartie, d'affaiblir celui-ci, en acceptant des réformes politiques d'envergure. Si l'aide sert à stabiliser les régimes, elle ne peut servir à les réformer, tout au plus peutelle concourir à les libéraliser. Mais libéraliser n'est pas démocratiser ${ }^{11}$. Libéraliser, c'est adopter une "stratégie de survie $»^{12}$, ou plus exactement - parce que le terme survie dénote un affaiblissement dont ne témoignent pas les faits - une stratégie de " consolidation de l'autoritarisme $»^{13}$. Cette consolidation consiste en un allégement plus ou moins significatif des contraintes pesant sur la sphère publique et sur l'activité des opposants, en échange de quoi ceux-ci acceptent de restreindre leur opposition au cadre légal restrictif mis en place par le régime.

\section{Les transitions accélérées}

8 L'approche par les "transitions accélérées » consiste, au contraire du gradualisme, à soutenir que le changement politique doit suivre un rythme rapide, d'abord fondé sur un accord politique impliquant des compromis entre des acteurs modérés, à l'instar de la transition espagnole qui a duré de novembre 1975 à décembre 1978. En ce sens, ce qui importe n'est plus la structure mais les acteurs. C'est ce que les théoriciens des transitions démocratiques nomment bien imprudemment l'«exceptionnalisme méthodologique $»^{14}$. À juste titre critiquée par Michel Dobry ${ }^{15}$, cette focalisation sur les effets conjoncturels d'actions contingentes comme moteur efficace de la transition vers la démocratie, découle du sentiment que le passage de l'autoritarisme à la démocratie se fait dans un temps ramassé, non par la modification des structures, mais par l'activité transformatrice d'acteurs occupant des positions stratégiques. Ce qui est donc propre à la théorie des transitions est l'accent qu'elles mettent sur la dynamique des conjonctures alors que le gradualisme met, lui, l'accent sur la modification des structures.

De ce point de vue, même la question de la primauté des réformes économiques sur les réformes politiques - ou l'inverse - se comprend, d'abord, comme un problème de conjoncture. A priori, il s'agit de l'idée selon laquelle la libéralisation de l'économie est un préalable nécessaire à la libéralisation politique. On retrouve la même idée dans l'approche gradualiste de la démocratisation qui s'inscrit ainsi dans la suite de la théorie des "prérequis ", initiée par Seymour Martin Lipset à la fin des années 195016. 
Toutefois, la libéralisation de l'économie n'est pas exactement entendue dans le même sens : s'agissant spécifiquement d'une transition, la question qui se pose n'est pas celle d'un préalable nécessaire, mais des obstacles que la réforme politique peut créer à la réforme économique et inversement. On considère que, dans certains cas, les réformes économiques empêchent ou ralentissent les réformes politiques et que, dans d'autres, les réformes économiques stabilisent les réformes politiques. C'est typiquement le cas des réformes économiques dans les pays de l'Est, qui viennent, juste après l'ouverture démocratique, priver les anciens dirigeants communistes de leurs assises économiques ${ }^{17}$. On observera, en outre, que dans le cas des transitions, l'ouverture est toujours politique et que c'est, en fait, à propos de la réussite de cette ouverture et de sa consolidation qu'intervient la variable économique, secondairement et de manière conjoncturelle.

\section{Dualisme sociologique}

10 Le gradualisme et la théorie des transitions accélérées relèvent ainsi, indubitablement, de deux sociologies fort différentes. Alors que le premier soutient (avec plus ou moins de fermeté) que les transformations des structures infra politiques - l'économie, la culture ou cet étrange objet qu'est le « capital social » - entraînent une transformation des structures politiques, la seconde cherche, quant à elle, à savoir ce qui va favoriser la réussite de la démocratie, une fois le mouvement donné. En fait, ni l'un ni l'autre ne disent exactement comment se fait le passage d'un état à un autre : on ne sait pas, avec le gradualisme, dans quelle situation précise advient la démocratie, et l'on ne sait pas, avec la théorie des transitions, quel est exactement le déclencheur de la transition. De ce point de vue, il ne paraît pas raisonnable, de prime abord, de faire confiance au gradualisme pour expliquer la sortie de l'autoritarisme, dans la mesure où il nous dit moins comment elle se fait qu'il ne prédit son arrivée. En effet, l'économie peut aller de mieux en mieux, du point de vue de la doctrine libérale, et l'autoritarisme se porter comme un charme. La Chine en offre un exemple et l'Inde un contre-exemple, puisque les faiblesses du développement n'y ont pas empêché l'établissement d'un État démocratique. On peut toutefois faire, à peu de choses près, le même constat en ce qui concerne la théorie des transitions : elle ne dit pas comment on en arrive là. Elle part d'une situation de crise du régime ${ }^{18}$. Elle dit comment on peut, avec plus ou moins de bonheur, se guider à travers les obstacles de la conjoncture pour réussir une transition. La question de l'apparition de la crise demeure toutefois irrésolue.

11 Certains régimes autoritaires décident, dans des circonstances favorables, de changer le paradigme en est la transition espagnole ${ }^{19}$. D'autres régimes autoritaires s'effondrent - le paradigme en est donné par nombre de pays de l'Est. Ces effondrements sont la conséquence d'évolutions internes confrontées à des faits de conjonctures, comme l'annonce du retrait des troupes soviétiques des pays de l'Est, en $1988^{20}$. Il ne faut donc pas abandonner les "structures ", quitte sans doute à les redéfinir, ainsi que le souligne Michel Dobry ${ }^{21}$, puisque c'est bien de leur nature et de leur agencement que découle la force des conjonctures. Prenons un exemple : si l'effondrement de la plupart des pays de l'Est fut si soudain, alors qu'il semblait que les régimes en place eussent la capacité de résister à bien des bouleversements, c'est que l'acquiescement des gouvernés et le dévouement d'une part des élites étaient relativement faibles, et que l'apparence de stabilité provenait de ce que chacun feignait d'adhérer à ce à quoi il se résignait. En ce 
sens, l'ordre provient de ce que Kuran nomme «falsification des préférences $»^{22}$. Dans ce type de situation, la structuration de la vie sociale est donc fondée sur une obédience partagée n'impliquant pas une identité de vue sur ce qu'il conviendrait de faire dans une situation différente. L'état stable de la structure est donc partiellement lié à la perception que les acteurs se font de la liberté d'action que leur accorde la conjoncture, telle du moins qu'ils peuvent l'imaginer en se fondant sur leurs attitudes respectives. Dans ce type de situations, une rupture simplement conjoncturelle de l'ordre ostensible peut donc entraîner des conséquences structurelles. Imaginons un régime autoritaire connu pour la promptitude de ses réactions répressives. Ce régime laisse, dans un contexte de relative pression internationale, se tenir une manifestation d'opposants. Pour le régime, il s'agit d'une concession (a priori) sans risque. Cependant, sa réputation de sévérité est si efficace que les opposants interprètent cette concession comme une expression de faiblesse. Ils s'engagent donc dans une conflictualité accrue, d'où ne peuvent s'ensuivre que deux lignes d'actions : soit un renforcement de la répression, soit un affaissement du régime, c'est-à-dire deux formes de restructuration de l'ordre politique. C'est par des mécanismes semblables que l'on peut s'expliquer la chute si rapide du régime de l'Allemagne de l'Est.

La conception des « structures " s'avère, ici, très différente de celle qui apparaît dans le gradualisme. En effet, il ne s'agit pas d'une distribution des ressources économiques et sociales à travers des strates de la population, qui par étapes, modifie le système social, mais des ressorts d'ordre conjoncturel de la structuration sociale. Ce qui importe, c'est la structuration découlant de l'entremise d'acteurs agissant collectivement dans (et conformément à) un contexte donné. On peut faire, à propos du gradualisme, la même remarque que firent les théoriciens de la «mobilisation des ressources " à propos de l'analyse des mouvements sociaux : les causes importent moins que le comment ${ }^{23}$. Or, le gradualisme demeure de l'ordre du pourquoi, que l'on explique par la croissance sans montrer comment la croissance modifie les choses. En fait, la différence fondamentale entre les deux approches, la gradualiste et la théorie des transitions accélérées, réside dans le fait que la première ne laisse aucune part aux acteurs et aux processus cognitifs qui interviennent dans la structuration sociale, alors que la seconde accorde une part importante, voire décisive, aux acteurs et aux processus cognitifs-réflexifs ${ }^{24}$. Ce qui me paraît plus déterminant est que l'approche gradualiste implique un moyen terme $e x-$ ante, alors que la seconde approche implique un court terme ex-post : elle intervient, en somme, après quelque chose qui a eu lieu et qui a affecté la structuration de la société. Les deux approches manquent donc le basculement à proprement parler. Certes, ce basculement peut être relaté ex-post, mais est-il prévisible ex-ante, peut-il être produit, et de quelle manière? On passe ainsi de la question du dualisme sociologique à l'adoption d'une stratégie mixte, c'est-à-dire que l'on quitte le point de vue de l'analyste pour celui de l'acteur.

\section{Stratégie mixte : l'exemple américain}

13 Du point de vue de l'acteur attaché aux transformations politiques, le gradualisme n'est pas satisfaisant, en premier lieu par la temporalité qu'il implique. On imagine aisément que la temporalité des transitions accélérées lui conviendrait mieux, mais elle implique, pour commencer le jeu, que quelque chose ait changé dans l'autoritarisme, c'est-à-dire que l'on soit - pour des raisons indéterminées a priori - entré dans une 
conjoncture fluide ${ }^{25}$. Si l'on se place dans la perspective des acteurs internes attachés aux transformations politiques et du point de vue des acteurs externes également partisans de celles-ci, la question se pose des conjonctures rendant possible une restructuration de l'ordre politique. Les partisans des transitions accélérées avancent deux types de facteurs : 1) l'effondrement du régime en place du fait de la difficulté qu'il éprouve à maintenir son organisation répressive, pour des raisons aussi bien internes qu'externes; 2) l'effondrement du régime du fait d'un dysfonctionnement majeur dans l'allocation (différentielle) des ressources, tant à ses partisans qu'au restant de la population ${ }^{26}$. S'agissant $d u$ monde arabe, la question du dysfonctionnement de l'allocation des ressources joue relativement peu, dans la mesure où aucun changement dramatique n'est venu mettre en cause les équilibres établis ${ }^{27}$. Reste, l'effondrement de la capacité répressive du régime. C'est généralement à cela que travaillent, avec plus moins de bonheur, les partisans des réformes. L'espoir de cet effondrement est fondé sur l'idée que l'organisation structurelle de l'autoritarisme (son apparence massive, si l'on veut) est en fait tributaire des dynamiques conjoncturelles, en d'autres termes que la stabilité n'est pas un état mais la reconduction indéfinie de conjonctures favorables, une équivoque plus ou moins durable fondée sur la falsification des préférences. Pratiquement, la question se pose donc des manières d'amener un affaiblissement des capacités répressives des régimes en place, seule stratégie apparemment possible pour parvenir à l'orée d'une transition. L'état des lieux et l'expérience montrant qu'il y avait peu à attendre d'un déclenchement interne, amené de manière gradualiste ${ }^{28}$, l'idée d'un déclenchement externe pouvait sembler tentante par référence aux conséquences de la déclaration de Gorbatchev en décembre 1988, annonçant le retrait des troupes soviétiques des pays de l'Est $^{29}$. Ce retrait indiquait qu'en cas de désordre ou de déstabilisation, les gouvernants des déjà anciens "pays frères" ne pourraient compter que sur eux-mêmes, ce qui provoqua une transformation des attentes des différentes acteurs. Les gouvernants, ou tout au moins une partie d'entre eux, pouvaient craindre que l'usage de la répression se fasse sans assurance, puisqu'il faudrait faire face à la fois à la population et à un environnement international hostile. En d'autres termes, la répression leur apparut potentiellement plus coûteuse et plus risquée que la conciliation. Il n'y a aucune raison d'imaginer que les opposants n'agirent pas en fonction des mêmes considérations : ils puisèrent une plus grande assurance pour eux-mêmes dans le manque d'assurance des gouvernants ${ }^{30}$.

Josep Colomer insiste sur l'importance de l'imitation dans les transitions démocratiques, c'est-à-dire sur le fait que la trame universelle des attitudes rationnelles (au sens, fautil le dire, de la rationalité limitée ${ }^{31}$ ) et des contraintes organisationnelles rendent envisageable de reproduire, dans d'autres lieux, les systèmes de causalité découlant des anticipations réflexives des acteurs qui aboutirent, en un précédent lieu, à la victoire de la démocratie ${ }^{32}$. On peut imaginer, dès lors, que la cascade démocratique qui suivit le retrait soviétique des pays de l'Est ait tout bonnement inspiré aux Américains une stratégie consistant à provoquer une cascade identique à partir de la démocratisation (espérée) de l'Irak ${ }^{33}$. Cependant, la différence notable entre le monde arabe et l'Europe de l'Est tient à ce que les régimes autoritaires y sont égocentrés, chacun possédant une structuration politique particulière dotée de ses propres contraintes organisationnelles ${ }^{34}$ et ne dépendant pas, pour sa survie, des régimes voisins. Cette fragmentation des organisations politiques rend a priori difficile un effet de contagion. Certes, on peut imaginer une dynamique d'imitation, au sens de Colomer, mais l'on ne 
saurait oublier que cette dynamique fut enclenchée, dans les pays de l'Est, par le retrait contraint d'un acteur pivotal ${ }^{35}$, l'Union soviétique. Ce retrait fut simultanément manifeste pour l'ensemble des acteurs de chaque transition démocratique et modifia largement leurs attentes, en leur donnant le sentiment sans précédent d'un risque (pour les gouvernants) et d'une opportunité politique ${ }^{36}$ (pour les opposants). Toutefois, une telle modification des attentes et des anticipations de chacun ne fut à ce point productive que parce que les différents régimes autoritaires en place étaient en quelque sorte extravertis, c'est-à-dire fondés sur une assurance extérieure - la présence soviétique - qui, soudain, faisait explicitement défaut. Certes, l'Irak de Saddam Hussein n'était en rien un acteur pivotal au Moyen-Orient, mais l'intervention américaine, elle, était à même de modifier les jeux d'anticipation et d'assurance des acteurs régionaux. On distinguera toutefois ce que Daniel Brumberg nomme les «stratégies de survie » des régimes autoritaires, des modifications dictées par l'aménagement de l'autoritarisme, en vue de créer des régimes "semi-autoritaires". De même les "événements critiques $»^{37}$ par lesquels s'amorce une transition démocratique sont à distinguer des stratégies de survie et des aménagements de l'autoritarisme, qui pour leur part relèvent de la simple libéralisation politique et non d'un processus de transition.

\section{Les évolutions du Proche-Orient et l'exception libanaise}

La logique d'une transition consécutive à l'action des États-Unis n'apparaît, pour l'instant, qu'au Liban. En effet, la proche présence américaine comme son hostilité déclarée au gouvernement syrien ont largement modifié la manière dont les opposants libanais au maintien d'une souveraineté limitée du Liban au profit des Syriens et de leurs affidés pouvaient évaluer les risques et les opportunités d'une action protestataire. À la suite de l'assassinat de Rafic Hariri, la situation de la Syrie fut si compromise, de manière si avérée, que les opposants purent à la fois manifester leurs sentiments et se coordonner. De vastes actions collectives devinrent possibles et les gouvernants durent, finalement, accepter la mise en place d'un gouvernement transitoire dirigé par Najib Mikati, un modéré (de leur bord). Nous sommes bien, ici, dans une situation où la neutralisation d'un acteur pivotal - la Syrie - constitue un événement critique pour un régime et enclenche une première phase de transition ${ }^{38}$. Tel n'est pas le cas de la réforme constitutionnelle égyptienne. L'élection du président de la République au suffrage universel direct relève bien plutôt d'une stratégie de libéralisation, c'est-à-dire d'une concession ne mettant pas en cause (même marginalement) la distribution du pouvoir, puisque, dans l'état actuel des choses, aucun candidat n'est en mesure de faire bonne figure dans une campagne présidentielle ni ne peut, a fortiori, espérer sérieusement mettre en difficulté le chef de l'État égyptien. On dira la même chose des élections municipales en Arabie saoudite, et de l'extension aux femmes du droit de vote au Koweï. De fait, ces divers changements paraissent s'inscrire dans la logique gradualiste de réforme et de développement global - celle présidant au projet du Grand Moyen-Orient ${ }^{39}$ - bien plus que dans la logique de transition. Ils n'enlèvent rien à la suprématie des gouvernants.

Si l'intervention des États-Unis en Irak a indirectement provoqué une neutralisation relative de la Syrie comme acteur déterminant de la vie politique libanaise, on ne saurait considérer a priori que les États-Unis avaient bien à l'esprit de neutraliser la 
Syrie afin de favoriser l'émancipation politique du Liban ${ }^{40}$. Ainsi que l'a remarqué Jon Elster, un résultat favorable à un acteur n'est pas nécessairement la conséquence d'une stratégie destinée à parvenir, en toute connaissance de cause, à ce résultat ${ }^{41}$. Elle peut être le résultat d'une action entreprise à d'autres fins. La séquence suivante apparaît donc comme la plus probable : 1) Les États-Unis décident de provoquer un « événement critique » en intervenant militairement en Irak, c'est-à-dire de modifier les jeux d'attentes et d'anticipation des gouvernants autoritaires comme des gouvernés; ils comptent explicitement sur un effet d'imitation, en mettant en place un gouvernement démocratique dans une région entièrement vouée à l'autoritarisme. 2) Cet espoir s'avère (au mieux) partiellement infondé, la création d'un État démocratique irakien étant mal aisée et, pour le moins, non exemplaire. 3) En revanche, la proximité américaine neutralise la Syrie et rend possible le développement d'un vaste mouvement social au Liban. Cette configuration, caractéristique de la phase initiale des transitions, n'a pas été reproduite ailleurs et n'est, d'ailleurs, probablement pas reproductible. Sa particularité l'explique aisément : elle est la seule où l'intervention américaine affecte directement un régime, en inhibant sa capacité répressive, parce qu'il est le seul où cette pression constitue une menace précise. Ce fut une chance pour le Liban. 4) En même temps, la vigueur de l'engagement américain constitue une menace diffuse pour ses alliés autoritaires, menace largement insuffisante pour les amener à des concessions significatives, mais suffisamment marquée pour les conduire à adopter de nouvelles politiques de libéralisation. Ces politiques de libéralisation s'inscrivent dans le cadre gradualiste. Elles ne surviennent pas à la suite d'un événement critique, mais tentent, au contraire, de se prémunir de sa venue. La stratégie américaine à l'origine de cette séquence est donc une stratégie mixte, dans la mesure où elle visait à produire des réactions en quelque sorte indéterminées, aussi bien chez les alliés de l'Amérique avec qui le conflit est quasiment inenvisageable que chez ses «adversaires » avec qui le conflit est envisageable. Ces réactions s'inscrivent, de facto, respectivement dans la logique gradualiste et dans la logique de transition.

\section{Le signal et l'événement critique}

Revenons à la théorie et au point de vue de l'analyste. Il est clair, désormais, qu'une analyse conduite uniquement en termes de transition accélérée ne peut rendre compte de la stratégie mixte des Américains, parce qu'elle se fonde seulement sur l'efficacité supposée d'un signal, sans entrer dans le détail des contextes. Elle peut en revanche rendre compte de l'inefficacité de cette stratégie, s'agissant des gouvernants alliés. En fait, ce qui fait la différence entre un signal déclenchant un événement critique et un signal déclenchant une simple politique de libéralisation tient en trois points : d'abord le contexte d'anticipation des conséquences de ce signal; puis l'apparition et l'élargissement croissant d'une fracture entre les performances des gouvernants et les attentes de la population ${ }^{42}$; enfin l'affaiblissement subséquent de la crédibilité du statu quo favorable au régime, de sorte qu'il suffit précisément d'un signal pour que les préférences déjà formées s'expriment. Si les gouvernés potentiellement opposants - en un mot les mécontents - sont persuadés, et se persuadent réciproquement, de la faible probabilité de la répression, les risques suscités par la manifestation de leurs véritables préférences leur sembleront relativement faibles, puisqu'ils auront la certitude d'avoir le nombre avec eux. 
18 Envisageons, maintenant, le cas de figure de l'Égypte ${ }^{43}$ par rapport à celui du Liban. Les gouvernants égyptiens n'ont pas à craindre une intervention américaine directe qu'elle prenne une forme militaire ou se limite à un embargo - visant à contrecarrer leur gestion répressive de l'opposition. Contrairement à la Syrie, perçue par Washington comme un adversaire déclaré, l'Égypte est un allié stratégique ${ }^{44}$. Cela est évident pour les gouvernants et pour la très grande majorité des opposants, de sorte que l'on ne peut sérieusement assimiler les diverses manifestations d'opposants aux prémices d'un plus vaste mouvement, impliquant une "cascade informationnelle » ${ }^{45}$. De plus, la crédibilité d'un "signal » entraînant un mouvement social dépend pour beaucoup de la crédibilité de ceux qui l'émettent. S'il parvient de groupes qui ne sont pas habitués à manifester leur opposition, il apparaîtra plus aisément aux autres groupes que leur perception de l'incurie du régime est une perception partagée. S'il ne parvient que de groupes plus ou moins habitués à manifester leur opposition, le signal n'aura qu'une faible portée et n'entraînera pas une agrégation suffisante pour constituer la masse critique à partir de laquelle les gouvernants se trouveront devant l'alternative de réprimer ou de céder ${ }^{46}$. C'est ce qui s'est passé au cours du premier semestre 2005 avec le mouvement «Kifaya», mouvement de protestation contre la prochaine candidature du président Moubarak à un sixième mandat et agrégeant divers opposants, des nassériens aux Frères musulmans, en passant par les militants de partis d'opposition dont les formations ne participent pas au mouvement en tant que telles ${ }^{47}$. Le régime n'a donc pas connu un "événement critique" au sens de la théorie transitologique. De leur côté, les Américains n'ont pas fait plus que ce qu'implique la doctrine gradualiste : exercer des pressions compatibles avec le maintien de relations stratégiques nécessaires. Le mouvement «Kifaya "n'a donc pas fait le «printemps arabe au Caire ». Un événement critique - si un tel événement devait advenir - serait bien plutôt lié à l'inéluctable succession présidentielle ${ }^{48}$ qu'à la modification des règles de désignation du chef de l'État.

19 En ce sens, si la clef d'une transition est un événement critique, les libéralisations pouvant (éventuellement) aboutir à une transition démocratique sont seulement les libéralisations qui succèdent à un événement critique et non les libéralisations qui tentent de s'en prémunir. Le signal américain, finalement assez comparable à la stratégie du coup de pied dans le réveil, n'a été un événement critique que pour l'organisation syrienne de l'autoritarisme au Liban. En Égypte, où le signal n'a fait que prendre la forme d'un surcroît de pression sur cette « course de mille pattes » qu'est le gradualisme, une telle opportunité n'est tout simplement pas envisageable. De ce point de vue, le gradualisme apparaît lié aux politiques de libéralisation prévenant les événements critiques et demeure la dominante des stratégies mixtes. Son fondement n'est pas dans le fait que l'on peut arriver à la démocratie de manière ordonnée sans faire subir de dommages directs aux régimes autoritaires, mais dans le fait que l'on ne peut, pour des raisons stratégiques, en user par trop cavalièrement avec eux. Le gradualisme est donc une vraie stratégie mais une théorie sociologique erronée. Ce n'est pas le cas de la théorie des transitions accélérées, puisqu'on peut, à partir de la modélisation qu'elle propose, distinguer les libéralisations sans transitions caractéristiques des politiques gradualistes, des libéralisations succédant à un événement critique, qui sont la première étape d'une transition. Toutefois, la théorie des transitions ne nous permet pas exactement de comprendre à quel moment survient un événement critique et sous l'effet de quelles évolutions. Sans doute trop centrée sur les négociations ex-post, elle ne décrit pas vraiment le jeu des anticipations, des 
préférences et des décisions qui déterminent ex-ante la survenance et la cristallisation d'un événement critique. L'ébranlement du régime par l'extérieur n'est possible que s'il a perdu sa crédibilité - à la fois comme dispensateur de ressources et comme organisation répressive - auprès d'une partie significative de la population. C'est donc la capacité du régime à maintenir ressources et répressions qu'il faut analyser. De ce point de vue, on ne saurait pas davantage suivre naïvement les travaux portant sur les transitions démocratiques dans les pays de l'Est. Le lieu compte, ainsi que le soulignait Valérie Bunce, en distinguant les transitions en Europe de l'Est des transitions en Europe du Sud ${ }^{49}$.

L'élargissement croissant d'une fracture entre les performances des gouvernants et les attentes de la population, qui fut à l'origine des «cascades informationnelles » et de l'effondrement des régimes à l'Est, n'est tout simplement pas à l'ordre du jour dans le monde arabe, quoiqu'il y paraisse. D'abord, parce que les régimes affichant des ambitions bien moins fortes, le différentiel entre les ambitions affichées et les réalisations risque moins d'apparaître comme un "signal ", entraînant les opposants modérés ou les citoyens déçus. Ensuite, parce que les politiques gradualistes, ironiquement soutenues par les adversaires prudents de l'autoritarisme, viennent apporter aux régimes les ressources nécessaires à la gestion de ce différentiel: des réformes politiques minimales, pour ne pas désespérer les opposants modérés, et des aides au développement pour ne pas désespérer ceux qui pensent, qu'à tout prendre, le statu quo est préférable à l'aventure. De la gestion de ces deux variables dépend le maintien des régimes autoritaires. Seul un dérèglement de cette gestion pourrait impulser une transition vers la démocratie. Mais il n'existe aucune théorie mécaniste d'un tel dérèglement.

\section{NOTES}

1. Sur l'aide conditionnelle, voir C. Santiso, «Promoting Democracy by Conditioning Aid? Towards a More Effective EU Development Assistance ", International Politik und Gesellschaft Online, $\mathrm{n}^{\circ}$ 3, 2000 (http: //festportail.fe.de). Voir aussi, S. Carapico, «Foreign Aid for Promoting Democracy in the Arab World ", Middle East Journal, vol. 56, n 3, 2002.

2. Par libéralisation politique, on entend autre chose, et surtout moins, que la démocratisation. La libéralisation désigne un desserrement de l'emprise du régime sur la vie publique. Du point de vue des gouvernants autoritaires, il ne s'agit pas d'un préalable à la démocratisation - c'est-à-dire la tenue d'élection libres, répétées à dates fixes et qui ne sont pas toujours remportées par les gouvernants. Voir, par exemple, la définition de J. A. Cheibub et A. Przeworski, «Democracy, Elections and Accountability for Economic Outcomes ", in A. Przeworski, S. C. Stokes et B. Manin, Democracy, Accountability and Representation, Cambridge, Cambridge University Press, 1999, pp. 222-223.

3. Voir par exemple B. Dillman, "Facing the Market in North Africa », The Middle East Journal, vol. 55, n 2, 2001, pp. 165-181 ; B. Dillman, «International Markets and Partial Economic Reform in North Africa », in R. Gillepsie et R. Young (dir.), The European Union and Democracy Promotion : the Case of North Africa, Londres, Frank Cass, 2002, pp. 234-249. 
4. Voir par exemple, B. Hibou, "Les marges de manœuvre d'un "bon élève" économique : la Tunisie de Ben Ali », Les Études du CÉRI, n 60, 1999.

5. Cela explique, par exemple, la mise en cause des « Middle Eastern studies » qui auraient failli à reconnaitre ce mal. Voir, par exemple, M. Kramer, Ivory Towers on Sand. The Failure of Middle Eastern Studies in America, Washington, Washington Institute for Near East Policy, 2001 et la critique bienvenue de ce point de vue: S. Heydemann, "Defending the Discipline ", Journal of Democracy, vol. 13, $\mathrm{n}^{\circ}$ 3, 2002.

6. R. Balme et B. Cautres, "La rationalité et les fondements sociologiques de la démocratie", L'Année sociologique, vol. 47, n² 2, 1997.

7. Pour une analyse critique de la stratégie gradualiste, voir T.Carothers, «Is Gradualism Possible ? Choosing a Strategy for Promoting Democracy in the Middle East », Working Papers, New York, Carnegie Endowment for International Peace, 2003 (Middle East Series, n 39).

8. A. de Tocqueville, De la démocratie en Amérique, II, II, VII.

9. Voir J.-N. Ferrié, « Les limites d'une démocratisation par la société civile en Afrique du Nord », Maghreb-Machrek, n 175, 2003.

10. Voir S. Carapico, «Foreign Aid for Promoting Democracy in the Arab World", Middle East Journal, vol. 56, $\mathrm{n}^{\circ}$ 3, 2002 et R. Youngs, « The European Union and Democracy in the Arab-Muslim World », CEPS Middle East et Euro-Med Project, Working paper n², Bruxelles, Centre for European Policy Studies, 2002.

11. Voir D. Brumberg, «Liberalization versus Democracy: Understanding Arab Political Reform », Working Papers, New York, Carnegie Endowment for International Peace, 2003 (Middle East Series, $\mathrm{n}^{\circ}$ 37).

12. Voir D. Brumberg, "Democratization in the Arab World? The Trap of Liberalized Autocracy », Journal of Democracy, vol. 13, $\mathrm{n}^{\circ}$ 4, 2002.

13. Comme le souligne M. Camau, "Remarques sur la consolidation autoritaires et ses limites", in A. Boutaleb, J.-N. Ferrié et B. Rey (coord.), L'Autoritarisme dans le monde arabe. Autour de Michel Camau et Luis Martinez, Cédéj, Le Caire (coll. « Débats »), 2005.

14. Voir G. Di Palma, To Craft Democraties, Berkeley University of California Press, 1990, cité et critiqué par M. Dobry, «Les voies incertaines de la transitologie. Choix stratégiques, séquences historiques, bifurcations et processus de path dependence ", Revue française de science politique, vol. 50, n $4-5,2000$, pp. 606 sqq.

15. Ibid.

16. Voir S. M. Lipset, «Some Social Requisistes of Democracy: Economic Development and Political Legitimacy », The American Political Science Review, vol. 53, n 1, 1959.

17. Voir V. Bunce, "Quand le lieu compte. Spécificités des passés autoritaires et réformes économiques dans les transitions à la démocratie ", Revue française de science politique, vol. 50, $\mathrm{n}^{\circ} 4-5,2000$ et «Rethinking Recent Democratisation. Lesson from the Postcommunist Experience », World Politics, vol. 55, janvier 2003.

18. Comme le remarque Ph. Schmitter, "Se déplaçant au Moyen-Orient et en Afrique du Nord, "transitologues" et "consolidologues" sont-ils toujours assurés de voyager en toute sécurité ?", Annuaire de l'Afrique du Nord 1999, Paris, CNRS Éditions, 2002, p. 17.

19. Voir J. Colomer, "Transition by Agreement : Modeling the Spanish Way ", American Political Science Review, vol. 85, $\mathrm{n}^{\circ} 4$ et Game Theory and Transition to Democracy: the Spanish Model, Aldershot, Edward Elgar, 1995.

20. Voir M. Dobry, «Les causalités de l'improbable et du probable: notes à propos des manifestations de 1989 en Europe centrale et orientale », Cultures et Conflits, n 17, 1995 ; T. Kuran, "The East European Revolution of 1989 : Is it surprising that we were surprised?» American Economic Review, vol. 81, $\mathrm{n}^{\circ}$ 2, 1991 et «Now Out of Never : the Element of Surpise in the East European Revolution of 1989 ", World Politics, vol. 44, n 1, 1991.

21. Voir M. Dobry, « Les voies incertaines de la transitologie... », art. cit., p. 609. 
22. C'est l'analyse de T. Kuran, «The East European Revolution of $1989 . .$. », art. cit. Ce mécanisme est développé dans T.Kuran, Private Truths, Public Lies. The Social Consequences of Preferences Falsification, Cambridge, Mass., Harvard University Press, 1995.

23. Voir J. MacCarthy et M. Zald, "Ressource Mobilization and Social Movments : a Partial Theory ", American Journal of Sociology, vol. 82, n 6, 1977.

24. Sur cette distinction, voir aussi J. M. Colomer, «Appendix A: Structural versus Strategic Approaches to Political Change", in J. M. Colomer, Strategic Transitions. Game Theory and Democratization, Baltimore, The Johns Hopkins University Press, 2000.

25. Voir M. Dobry, Sociologie des crises politiques, Paris, Presses de Sciences-Po, 1992.

26. Voir J. M. Colomer, Strategic Transitions... op. cit., pp. 4-5, et B. Bueno de Mesquito, J. D. Morrow, R. M. Siverson et A. Smith, «Policy Failure and Political Survival : the Contribution of Political Instituion », The Journal of Conflict Resolution, vol. 43, n² 2, 1999.

27. On ne retiendra pas les rapports du PNUD sur le développement humain : la question n'est pas de savoir si objectivement les dirigeants arabes ont failli à assurer le bien être de leurs populations, la question est de savoir si subjectivement ce constat peut déterminer une mise en cause des régimes en place. Or ce constant n'embraye pas sur la population et ne s'inscrit dans un aucun mouvement social. Produit par des intellectuels et des chercheurs arabes, il est d'abord destiné à des organismes internationaux et aux gouvernements "occidentaux ». Il peut servir à accréditer l'idée, auprès de ce public, que les réformes sont nécessaires mais non à les promouvoir.

28. C'est-à-dire par le développement de l'économie de marché et de la société civile. Voir J.N. Ferrié, « Les limites d'une démocratisation... », art. cit.

29. Voir J. M. Colomer, Strategic Transitions..., op. cit.

30. Voir, par exemple, l'analyse de S. Lohman, «The Dynamics of Informational Cascades : the Monday Desmonstrations in Leipzig, East Germany 1989-91 », World Politics, vol. 47, n 1, 1994.

31. Dans l'acception de H. A. Simon, Models of Bounded Rationality, Cambridge, Mass, MIT Press, 1982 ; voir aussi J. Elster, Le Laboureur et ses enfants. Deux essais sur les limites de la rationalité, Paris, Éditions de Minuit, 1987.

32. Voir J. M. Colomer, Strategic Transitions..., op. cit. p. 5.

33. Voir T. Carothers et M. Ottaway, Uncharted Journey: Promoting Democracy in the Middle East, New York, Carnegie Endowment for International Peace, 2005, pp. 4 sqq.

34. Sur les différentes organisations des régimes autoritaires arabes et les contraintes propres de chacune d'entre elles, voir N. Bernard-Maugiron et J.-N. Ferrié (dir.), Architectures constitutionnelles et démocratisation dans le Monde arabe, Égypte-Monde arabe, 3e série, $\mathrm{n}^{\circ} 2$.

35. On entend par là un acteur déterminant dans les jeux d'anticipations et de coordination (ou de non-coordination) d'un ensemble d'acteurs, qui considèrent son soutien, sa défection ou son opposition comme une variable dominante dans l'établissement et la conduite de leurs stratégies. 36. Suivant l'expression de S. Tarrow, Democracy and Disorder : Protest and Politics in Italy 1965-1975, Oxford, Clarendon Press, 1989.

37. Voir J. M. Colomer, Strategic Transitions..., op. cit., ch. 2 (notamment figure 2.2).

38. Dont rien n'indique ex-ante qu'elle sera suivie d'une deuxième, mais dont la deuxième ne pourra advenir que si la précédente est établie et dont elle constituera donc le préalable nécessaire ex-post. Cette formulation rétrospective devrait éviter l'erreur téléologique plus ou moins implicitement présente dans bien des approches transitologiques. Voir M. Dobry, «Les voies incertaines de la transitologie... », art. cit., pp. 592-593 et M. Camau, «La transitologie à l'épreuve du Moyen-Orient et de l'Afrique du Nord", Annuaire de l'Afrique du Nord 1999, Paris, CNRS Éditions, 2002.

39. Voir, par exemple, "What Strategy for the Greater Middle East ? ", ESF Working Paper, $\mathrm{n}^{\circ} 15$, Bruxelles-Londres Centre for European Policy Studies-The International Institute for Strategic Studies, 2003. 
40. Ne serait-ce que parce que la capacité de l'opposition libanaise à réagir à cette opportunité ne découle pas de l'action américaine. Cette capacité possède sa propre dynamique et ses propres jeux d'acteurs. Voir l'analyse de Samir Kassir : «Des esprits chagrins diront que la fin de la tutelle syrienne au Liban est le résultat des pressions internationales, concrétisées par la résolution 1559. Voire. C'est oublier que toutes les révolutions dans l'histoire ont été favorisées par la conjoncture régionale ou internationale. C'est ignorer que la mobilisation des puissances internationales n'a été rendue possible que par la montée des résistances au sein de la société libanaise contre la normalisation de l'arbitraire baassiste, et plus particulièrement contre la décision du président syrien Bachar Al-Assad de faire proroger par un Parlement aux ordres le mandat du président libanais Émile Lahoud, en dépit de l'impopularité de ce dernier et du simple bon sens » («Un printemps arabe », La revue d'Égypte, $\left.n^{\circ} 16,2005\right)$.

41. Voir J. Elster, Le Laboureur et ses enfants... op. cit.

42. Sur l'idée d'un différentiel entre les perceptions que les acteurs ont de leur situation et sur le jugement qu'ils portent sur les politiques publiques comme cause de l'engagement dans un mouvement social, voir J. DeNardo, Power in Numbers, Princeton, Princeton University Press, 1985. 43. Ce cas est développé dans J.-N. Ferrié, «L'Égypte : des réformes nécessaires et périlleuses », Questions internationales, $\mathrm{n}^{\circ} 15$ (à paraître).

44. Sans doute moins important qu'avant mais non négligeable, voir J.-N. Ferrié, « L’Égypte : des réformes nécessaires... », art. cit.

45. On appelle " cascade informationnelle » une relation dans laquelle le choix de A se modèle sur le choix de B et celui de C sur celui de A et de B. La raison de choisir une option plutôt qu'une autre ne résulte pas d'une préférence propre mais de l'influence qu'exercent les choix précédents. Ce phénomène a été décrit à propos de l'Allemagne de l'Est par S. Lohman, «The Dynamics of Informational Cascades... ", art. cit. Voir également S. Bikhchandani, D. Hirshleifer, I. Welch, «Learning from the Behavior of Others: Conformity, Fads, and Informational Cascades ", Journal of Economic Perspectives, vol. 12, $\mathrm{n}^{\circ}$ 3, 1998.

46. Sur la faible crédibilité de l'action des opposants résolus par rapport à la crédibilité d'une amorce par les opposants modérés, voir S.Lohmann, «The Dynamics of Informational Casacades... », art. cit., pp. 49 sqq. ainsi que S. Lohmann, "A Signaling Model of Informative and Manipulative Political Action ", American Political Science Review, vol. 83, n² 2, 1993. Sur la question de la masse critique, voir J. DeNardo, The Power of Numbers, op. cit.

47. Voir B. Rey, «Entre nouvelles formes de mobilisations et gestion étatique. L'opposition égyptienne en 2004 », in F. Kohstall (coord.), L'Égypte dans l'année. Chronique politique 2004, Le Caire, Cédéj, 2005.

48. Voir C. Hassabo, "Gamal Moubarak au centre du pouvoir : une succession achevée ? ", dans

F. Kohstall, (coord.), L'Égypte dans l'année... op. cit.

49. V. Bunce, « Quand le lieu compte... », art. cit.

\section{RÉSUMÉS}

L'article soutient que la conception gradualiste des réformes dans le monde arabe est une erreur sociologique et une erreur pratique. Il montre, au contraire, que la théorie des transitions est le point de départ d'une description analytique du changement politique. Les actions de la société civile ne peuvent être un substitut au changement politique lié à un évènement critique ouvrant 
vers une transition accélérée. De façon contrastée, les développements récents dans le monde arabe (Liban, Egypte) illustrent l'argumentaire ici défendu.

The paper argues that the gradualist conception of reform in the Arab world is a sociological and practical fallacy. It shows, on the contrary, that the theory of transitions is the starting point of an analytical description of the political change. Civil society movements are not a substitute for political change induced by a critical event which opens the way to an accelerated transition.. Contrasted recent developments in Liban and Egypt illustrate the point.

\section{INDEX}

Keywords : Arab World, critical event, gradualism, reform, transition Index géographique : monde arabe

Mots-clés : événement critique, gradualisme, monde arabe, réforme, transition

\section{AUTEUR}

\section{JEAN-NOËL FERRIÉ}

Centre d'études et de documentation économiques, juridiques et sociales (CEDEJ), Le Caire.

Docteur en science politique (Institut d'études politiques d'Aix-en-Provence) et habilité à diriger des recherches (Institut d'études politiques de Paris), Jean-Noël Ferrié est chercheur au CÉDÉJ du Caire. Il y a codirigé le programme : « Parlement et autoritarisme dans le monde arabe ». Il a été rédacteur en chef de l'Annuaire de l'Afrique $d u$ Nord (CNRS Éditions) et d'Égypte-Monde arabe, et a enseigné la science politique à l'Université du Caire et à l'Université Saint-Joseph, à Beyrouth. Il est membre du Centre de science politique comparative de l'Institut d'études politiques d'Aix-en-Provence. Il est l'auteur de Le Régime de la civilité en Égypte, paru chez CNRS Éditions, et a codirigé Dispositifs autoritaires et dispositifs démocratiques en Afrique du Nord, chez le même éditeur. ferrie@link.net 